\title{
Terra firma forme dermatosis and plica neuropathica - case report
}

\section{Rakesh Bharti}

Department of Dermatology, Bharti Dermacare and Research Centre, Amritsar, Punjab, India

Corresponding author: Dr. Rakesh Bharti, E-mail: rakeshbharti1@gmail.com

\begin{abstract}
Although Dirty Duncan Dermatosis and Plica Neuropathica are two different entities. Yet they have a common denominator of disturbed state of mind. Presenting here a case, who suffered from both, but forty years apart and with disturbed state of mind both times.
\end{abstract}

Key words: Terra firma-forme; Duncan dermatosis; Dermatitis neglecta

\section{INTRODUCTION}

Duncan first described this condition,less than thirty years ago [1]. The addition of words "Dirty Dermatosis" gives a feeling that this may be related with not so good hygiene of a person. TFFD however is distinguished from Dermatosis Neglecta arbitrarily by presence of adequate hygiene $[2,3]$.

Plica neuropathica, also called plica polonica, felting or bird's nest hair, was first described by Le Page in 1884It too presents as a compact mass of scalp hair with irregular twists and irreversibly entangled plaits that form a firm to hard impenetrable mass of keratin cemented together with dirt and exudates. it has been attributed to longitudinal splitting or weathering of hair shaft due to vigorous friction and frequent use of harsh shampoos and harsh cleansers; and/or due to keeping of long hair with poor hair care or its neglect. This entity has been also found to be more frequent among psychologically disturbed women due to the repeated manipulation of the hair [4-8].

Do the two conditions have anything in common.

This is a case report just as to create awareness about the TFFDso as to facilitate prompt diagnosis,avoid unnecessary invasive investigations (biopsy) and endocrine evaluation and also to give readers a food for thought-thought of linkage of two disorders in mind.

\section{CASE REPORT}

53 year old man presented with four weeks or so history of an asymptomatic brown rash on thighs, and legs. The patient assured daily shower with good quality soap and was not having any bad body odor at all. He gave the history of some disturbed state of mind due to his son's failure to get a VISA. On further interrogation, he revealed history of some hair dreadlocks in childhood, just like Indian sadhus, after his failure in a class (suggestive of mental stress) He remembered that he has to get his head shaved due to those stranded hair in childhood.

Physical examination revealed palpable, papillomatous blackish plaques on both thighs and legs. The examination also revealed that he is a hairy guy. Suspecting TFFD, the black pigmentation was tried to be rubbed off with spirit, but to limited success. The patient was then asked to go home, take a shower and use a hard scrub. Patient returned after about an hour,with no change in the clinical picture. At this juncture then, isopropyl 


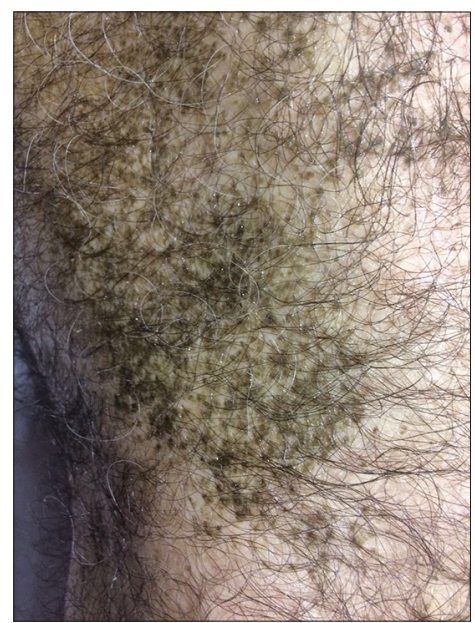

Figure 1: Before cleaning with Isopropyl alcohol.

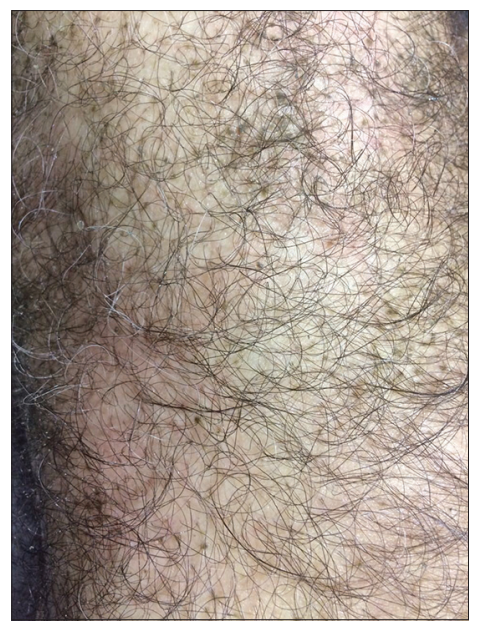

Figure 2: After cleaning with Isopropyl alcohol.

alcohol was procured from a nearby laboratory and a sample area could then be completely rubbed off blackish pigmentation (Figs. 1 and 2). Patient was sent off with the instructions about the method of cleaning with Isopropyl alcohol, a session of counseling to cope up with his mental tension and prescription of clonazepam $0.25 \mathrm{mg}$ at bed time for two weeks.

\section{DISCUSSION}

Terra firma-forme dermatosis derives its name from the: Latin phrase terra-firma meaning dry land (dirt). The condition was first described by Duncan and thus also known as Duncan's dirty dermatosis, so as to honor the physician first describing it. Terra firma-forme dermatosis is characterized by brown, dirt-like discoloration that cannot be removed by bathing with water or rubbed off with routine detergent soap. It has most often been seen in children, but it has also been described in adults. The condition most often involves the neck and trunk but has also been reported on the scalp] and the pubic region.In my case it involved both thighs and legs.

The cause of the condition remains unknown. The condition, however may look like Acanthosis nigricans, lichen amyloidosis, confluent and reticulated papillomatosis or dermatitis neglecta even. If suspected rubbing with isopropyl alcohol with some persistant pressure, dirt my come off, as it occurred in my case. This simple trick then, can save the patient from undergoing invasive investigations like biopsy and detailed endocrinological blood tests [2,3].

Plica neuropathica first described by Le Page in 1884 was attributed to the strange occurrence to nerve force by him but was considered a "visitation from God' by the parents of his case, a 17 year old girl $[4,5]$. This condition is also common among Indian Sadhus (ascetics), who do not take care of their hair and don't trim them. People have entangled plaits that form firm to hard impenetrable mass of keratin cemented together with exudates and dirt. The exact aetiopathogenesis of this condition is not known. It has, however, been attributed to longitudinal splitting or weathering of hair shaft due to friction, frequent use of shampoo and or keeping long hair with poor hair care or its neglect [6,7]. This entity has also been found commonly in psychologically disturbed persons due to repeated manipulation of hair $[5,8]$. In the present case of TFFD, patient gives the history of Plica neuropathica in childhood and also history of some mental disturbances at present. This makes me think of a common linkage of physical and mental status of TFFD. More insight, however is needed.

\section{CONCLUSION}

Although Terra Firma -forme Dermatosis and Plica neuropathica appear to be two distinct entities altogether, yet the occurrence of two in the same person (separated by forty years) with disturbed state of mind, failure in exam in childhood and failure of his child in later life, gives us a food for thinking. Also, considering the condition by an alert dermatologist can save the patient from invasive and elaborate investigations.

\section{REFERENCES}

1. Duncan WC, Tschen JA, Knox JM. Terra firma-form dermatosis. Arch Dermatol. 1987:123:567-9.

2. O'Brien TJ, Hall AP. Terra firma-forme derematosis. Australas J 
www.odermatol.com

Dermatol. 1997:38:163-4.

3. Raveh T, Gilead LT, Wexler MR. Terra firma-forme dermatosis. Ann Plast Surg. 1997:39:542-5.

4. Ghodake NB, Singh N, Thappa DM. Plica neuropathica (polonica):clinical and dermascopic features. Indian J Dermatol Venereol Leprol. 2013:79:269.

5. Kumar PN, Antony B, Chakravarthy A, Koyamu AM. Plica neuropathica(polonica) in schizophrenia-a case report and review literature. Indian J Psychiatry. 2001:43:281-3.

6. Kanwar AJ, De D. Plica neuropathica in a 2 year old boy. Int.J Dermatol. 2007:46:410-1.
7. Khare AK. Plica neuropathica. Indian J Dermatol Venereol Leprol. 1985:51:178-9.

8. Bharti R, Singh HP. Pimozide treatment of plica neuropathica. Indian J Dermatol Venereol Leprol, 1994;60:101-2.

Copyright by Rakesh Bharti. This is an open access article distributed under the terms of the Creative Commons Attribution License, which permits unrestricted use, distribution, and reproduction in any medium, provided the original author and source are credited.

Source of Support: Nil, Conflict of Interest: None declared. 